\title{
EQUIVARIANT FIBRATIONS AND TRANSFER
}

BY

STEFAN WANER

\begin{abstract}
The basic properties of equivariant fibrations are described, including an equivariant version of the Dold Theorem. The foundations of equivariant stable homotopy theory are described, and the theory of equivariant transfer is developed.
\end{abstract}

0. Introduction. The purpose of this paper is to set forth an account of the basic properties of equivariant fibrations, equivariant stable homotopy theory, and the equivariant transfer homomorphism (à la Becker-Gottlieb [Be1]). This will provide the necessary background for the classification of equivariant fibrations [Wa2] and for further results in stable equivariant homotopy theory ([Wa3], [MHW] and [Wa4]).

The paper is divided as follows:

1. Equivariant fibrations

2. Equivariant quasifibrations

3. Equivariant duality and stable homotopy

4. Equivariant fiberwise duality

5. Equivariant transfer

6. Equivariant cellular theory and fibrations

In $\$ \S 1$ and 2 , we establish an equivariant "Dold $\bmod 1$ " Theorem (the $\bmod k$ version is discussed in [Wa4]) and the Dold-Thom Theorem for quasifibrations, while the theory of the transfer is presented in $\$ \S 3-5$. Finally, $\$ 6$ gives conditions under which the total space of an equivariant fibration has the $G$-homotopy type of a $G-C W$ complex (as in [St1] and [Mi1]).

Our development of stable equivariant homotopy theory follows May [Ma1], and the correct indexing system of $G$-modules is described here and shown to be equivalent to various other systems which appear in the literature.

$G$ Q will denote the category of (unbased) $G$-spaces and continuous maps. (We require all spaces to be weak Hausdorff compactly generated [Ma1, §III].)

Throughout, $G$ will be assumed to be a compact Lie group. The relevant $G$-homotopy theory is discussed in [Wa1].

Received by the editors September 7, 1978.

AMS (MOS) subject classifications (1970). Primary 54H15.

Key words and phrases. G-fibrations, fiberwise, equivalence, stable G-equivalence, isotopy subgroup, transfer, fiberwise $G$-duality, equivariant cohomology. 


\section{Equivariant fibrations.}

Definition 1.1. By a $G$-space $p$ over $B$, we shall mean a map $p: E \rightarrow B$ in $G \mathscr{U}$. If $p$ and $q$ are $G$-spaces, then a morphism of $G$-spaces is a pair of $G$-maps $f$ and $f^{\prime}$ making the following diagram commute:

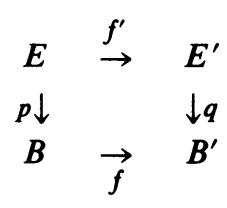

When $B=B^{\prime}$, we shall understand that $f$ is to be the identity map of $B$.

By a based $G$-space $p$ over $B$, we shall mean a $G$-space $p$ over $B$ together with a $G$-section $s$ of $p$ which is a fiberwise $G$-cofibration. Morphisms of based $G$-spaces are required to preserve the section.

Definition 1.2. An equivariant fibration over $B$ is a $G$-space $p$ over $B$ which satisfies the $G$-covering homotopy property ( $G$-CHP). Here, all homotopies are required to be through $G$-maps. (See [Wa1] for an account of $G$-homotopy theory.) A based fibration is defined analogously. (All homotopies are required to be through section preserving maps.)

Most of the discussion to follow is restricted to unbased $G$-fibrations, with remarks to indicate how to deal with the based variant where this is not straightforward. The following is a useful example of an equivariant fibration:

Definition 1.3. Let $F$ be a space. We shall say that a (based) $G$-fibration $p$ : $E \rightarrow B$ is a $G$-fibration with fiber $F$ if for each $b$ in $B$ there is some action of $G_{b}$ on $F$ such that $p^{-1}(b)$ is $G_{b}$-homotopy equivalent to $F$ with respect to the given action.

In general, the fixed point sets of $B$ may be disconnected, so that not all $G$-fibrations satisfy the above requirement. However, the interesting examples of $G$-fibrations are of such a nature.

Following is a necessary and sufficient condition for a $G$-fibration to be a $G$-fibration with fiber $F$ :

Proposition 1.4. $A$ G-fibration $p: E \rightarrow B$ is a fibration with fiber $F$ if and only if we can find a set $\left\{Q_{\alpha}\right\}$ of orbits in $B$ such that the inclusion $\amalg_{\alpha} Q_{\alpha} \rightarrow B$ induces $a$ surjection of all zeroth equivariant homotopy groups (see [Wa1]) and such that $p \mid p^{-1}\left(Q_{\alpha}\right)$ is a G-fibration with fiber $F$.

The proof is an immediate consequence of the G-CHP. (In [Wa2], these fibrations are referred to as $(G \mathscr{F} \mathscr{W}, F)$ fibrations.)

Of course, it follows that equivariant bundles are equivariant fibrations with fiber $F$, once we accept the existence of the $G$-CHP for $G$-bundles.

The following result is an immediate consequence of the $G$-CHP and gives us the expected long-exact fibration sequences (see [Wa1] for a discussion of $G$-homotopy groups).

Proposition 1.5. Let $p: E \rightarrow B$ be a G-fibration, then

$$
p_{*}: \pi_{n}^{H}\left(E, F_{b} ; G_{b}\right) \rightarrow \pi_{n}^{H}\left(B, b ; G_{b}\right)
$$


is an isomorphism of $G_{b}$ homotopy groups, for each $H<G_{b}$ and for $\pi_{n}^{H}\left(E, F_{b} ; G_{b}\right)$ nonempty. (Since $F_{b}$ is not G-invariant in general, it is meaningless to take G-homotopy groups.)

The requirement that $\pi_{n}^{H}\left(E, F ; G_{b}\right)$ be nonempty proves restrictive when we wish to appeal to the five-lemma when comparing the bases or total spaces of two $G$-fibratons. It is not, however, restrictive when we wish to compare corresponding fibers.

We have assumed, implicitly, the following consistency statement in deducing Proposition 1.5.

Proposition 1.6. Let $p: E \rightarrow B$ be a G-fibration, and let $H<G$ be a closed subgroup. Then $p: E \rightarrow B$ is an $H$-fibration.

Proof. We may convert an $H$-lifting problem to a $G$-lifting problem by considering the following diagram:

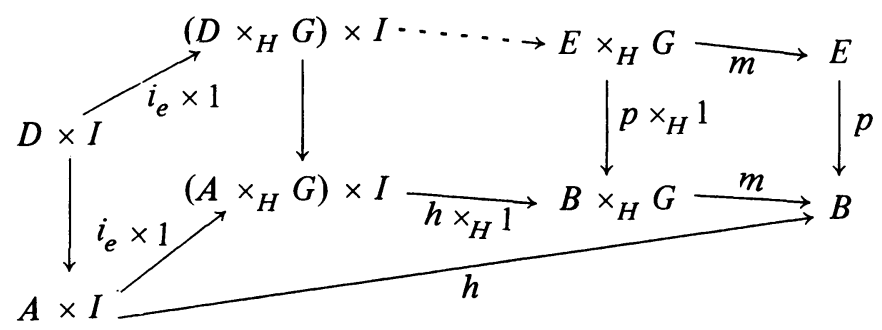

where all the squares are in $G \mathscr{Q}$ except for the left-hand one, which is in $H \mathscr{U}$ and $m$ is obtained from the $G$-action, while $i_{e}$ is inclusion $a \mapsto(a, e)$.

(This can also be proved using the $G$-gamma construction as discussed in [Wa3].)

The following material relates local fiberwise homotopy equivalence to its global counterpart.

Definition 1.7. Suppose that $p$ and $q$ are $G$-spaces over $B$ and that $g: p \rightarrow q$ is a morphism of $G$-spaces over $B$. A $G$-homotopy section of $g$ is a pair $\left(g^{\prime}, H\right)$ where $g^{\prime}$ : $p \rightarrow q$ and $H$ is a $G$-homotopy $g g^{\prime} \simeq 1$ (the notion of a $G$-homotopy of morphisms of $G$-spaces being the obvious one).

Lemma 1.8 (Section Extension Property). Suppose that $\phi: B \rightarrow I$ is a G-map and that $g: p \rightarrow q$ is a morphism of $G$-spaces over $B$ such that the part of $g$ over $\phi^{-1}(0,1]$ has a G-homotopy section $(f, K)$. Then there is a G-homotopy section $(\bar{f}, \bar{K})$ of $g$ agreeing with $(f, K)$ over $\phi^{-1}(1)$ whenever $g$ is a G-homotopy equivalence over $B$.

The proof in [Ma2, §1.4] goes over to the equivariant case verbatim; we merely check that all the maps constructed are equivariant.

By a $G$-cover, we shall mean a cover by invariant sets.

Definition 1.9. A $G$-cover $e$ of $B$ is numerable if it is locally finite and if for each $U \in \mathcal{C}$ there is a $G$-map $\lambda_{U}: B \rightarrow I$ with $U=\lambda_{U}^{-1}(0,1]$. 
Proposition 1.10. Suppose that $p: D \rightarrow B$ and $q: E \rightarrow B$ are $G$-spaces over $B$ with $g: D \rightarrow E$ a $G$-map over $B$ such that $g \mid p^{-1}(U)$ is a G-homotopy equivalence over $U$ for each $U$ in some numerable cover $\mathcal{C}$ of $B$. Then $g$ is a $G$-homotopy equivalence over $B$.

This follows by the same Zorn's lemma argument as that of [Ma2, §1.5].

Recall that a tube in a $G$-space $X$ is a $G$-subspace of the form $U \simeq V \times{ }_{H} G$ where $V$ is some $H$-invariant subspace of $U$, and $H$ is the isotropy subgroup of some point $v$ in $V$. The orbit $v G$ will be called the central orbit of $U$.

We have the following generalization of a theorem of Dold.

TheORem 1.11 (G-DoLD). Suppose that $B$ has a numerable G-cover $\mathcal{C}$ of open tubes which deform equivariantly to specified central orbits and that $g: p \rightarrow q$ is a map of $G$-fibrations over $B$ which restricts to an equivariant homotopy equivalence on each fiber. Then $\mathrm{g}$ is a fiberwise G-homotopy equivalence.

Proof. For each $U$ in $\mathcal{C}$, choose a $G$-homotopy $h_{U}: U \times I \rightarrow U$ which contracts the tube $U$ to the specified orbit $Q_{U}$. We then have the following commutative diagram:

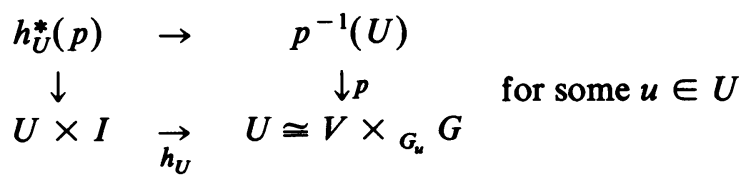

as well as a $G$-homotopy equivalence $h_{U}^{*}(p)\left|U \times\{0\} \simeq h_{U}^{*}(p)\right| U \times\{1\}$ over $U$; (one checks that the results in [Ma2, \$\$2.2-2.5] go over to the equivariant case with no change, the homotopies being patched by successive applications of the G-CHP to pass from one to another).

Abbreviating $h_{U}^{*}(p) \mid U \times\{0\}$ and $h_{U}^{*}(p) \mid U \times\{1\}$ to $D_{0}$ and $D_{1}$ respectively, we have $D_{0} \simeq p^{-1}(U)$ and $D_{1} \simeq\left(V \times F_{u}\right) \times{ }_{G_{u}} G$, where $G_{u}$ acts diagonally on $V \times$ $F_{u}=V \times p^{-1}(u)$. We have the same situation in $q: E \rightarrow B$, and we obtain spaces $E_{0}$ and $E_{1}$ corresponding to $D_{0}$ and $D_{1}$ respectively.

We therefore have the following diagram in which all the triangles are $G$-commutative:

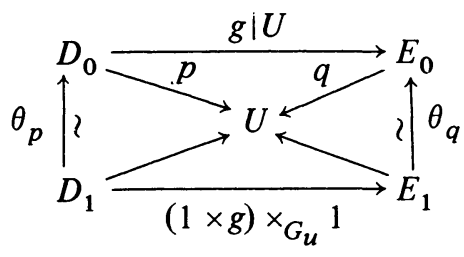

Here $\theta_{p}$ and $\theta_{q}$ are the natural maps, and the unlabelled arrows are the obvious maps. It thus suffices to show that the whole diagram $G$-homotopy commutes over $U$, by Proposition 2.1.6. But both $g \mid U \circ \theta_{p}$ and $\theta_{q} \circ(1 \times g) \times{ }_{G_{w}} 1$ are final lifts for the diagram: 


$$
\begin{array}{ccc}
\left(V \times F_{u}\right) \times{ }_{G_{u}} G=D_{1} \times I & \stackrel{H_{u}}{\rightarrow} & F_{0} \\
\downarrow & & \downarrow q \\
U \times I & \overrightarrow{\tilde{h}_{U}} & U
\end{array}
$$

where $H_{U}(((v, f), s), 0)=((u, g(f)), s) \in\left(\{u\} \times F_{u}\right) \times{ }_{G_{u}} G \subset E_{0}$ and $\tilde{h}_{U}(x, t)=$ $h_{U}(x, 1-t)$.

2. Quasifibrations and gammafication. We now discuss $G$-quasifibrations. Recall the definition of equivariant homotopy groups and of $G$ - $n$-equivalence from [Wa1].

Definition 2.1. A map $p: E \rightarrow B$ in $G Q$ is said to be a $G$-quasifibration if

$$
p_{*}: \pi_{n}^{H}\left(E, F_{b} ; \phi ; G_{b}\right) \rightarrow \pi_{n}^{H}\left(b, b, p \phi ; G_{b}\right)
$$

is an isomorphism for each $b$ in $B$, for each closed subgroup $H$ of $G_{b}$ and for each $G_{b} \operatorname{map} \phi: G_{b} / H \rightarrow F_{b}$. (This is equivalent to the result of 1.3.) Here, and henceforth, $G_{b}$ denotes the isotropy subgroup of $b$.

Observe that this is equivalent to the requirement that $p p_{n}: \pi_{n}^{H}\left(E, F_{b} G, \phi\right) \rightarrow$ $\pi_{n}^{H}(B, b G, p \phi)$ be an isomorphism, since $G \times{ }_{G_{b}} G_{b} / H \simeq G / H$ where $F_{b} G$ is the "saturation," $\left\{f \cdot g \mid f \in F_{b}, g \in G\right\}$ of $F_{b}$ and $b G$ is the orbit of $b$.

Definition 2.2. Suppose that $p: E \rightarrow B$ is in $G \mathcal{Q}$, and that $U \subset B$ is an equivariant subspace such that $p \mid: p^{-1}(U) \rightarrow U$ is a $G$-quasifibration. We then say that $U$ is a distinguished subset of $B$.

We now develop the excisive theory of $G$-quasifibrations according to the nonequivariant example.

Lemma 2.3. Suppose that $p: E \rightarrow B$ is distinguished on $A \subset B$, and that there are $G$-homotopies $h_{t}: B \rightarrow B$ and $H_{t}: E \rightarrow E$ such that:

(a) $h_{0}=1, h_{t}(A) \subset A$ and $h_{1}(B) \subset A$,

(b) $H_{0}=1, H_{t}\left(p^{-1}(A)\right) \subset p^{-1}(A)$, and $H_{1}$ covers $h_{1}$,

(c) $H_{1}: p^{-1}(b) \rightarrow p^{-1}\left(h_{1}(b)\right)$ is a weak $G_{b}$-equivalence.

Then $p$ is a $G$-quasifibration.

Proof. The following diagram commutes in $K \mathscr{Q}$, where $K=G_{b}$ :

$$
\begin{array}{ccc}
\left(E, F_{b}\right) & \vec{H}_{1} & \left(p^{-1}(A), F_{h_{1}(b)}\right) \\
\downarrow p & & p \downarrow \\
(B, b) & \stackrel{h_{1}}{\rightarrow} & \left(A, h_{1}(b)\right)
\end{array}
$$

Passing to homotopy groups then gives the diagram:

$$
\begin{array}{ccc}
\pi_{n}^{K^{\prime}}\left(E, F_{b}, \phi ; K\right) & \vec{H}_{1_{*}} & \pi_{n}^{K^{\prime}}\left(p^{-1}(A), F_{h_{1}(b)}, H_{1} \phi ; K\right) \\
\downarrow & & p_{*} \downarrow \\
\pi_{n}^{K^{\prime}}(B, b, p \phi ; K) & \stackrel{h_{1 *}}{\rightarrow} & \pi_{n}^{K^{\prime}}\left(A, h_{1}(b), h_{1} \phi ; K\right)
\end{array}
$$

for $K$-maps $\phi: K / K^{\prime} \rightarrow F_{b}$ and subgroups $K^{\prime}$ of $K$. 
By the hypothesis, the right-hand vertical map is an isomorphism. It thus suffices to show that $h_{1}$ and $H_{1}$ are isomorphisms. Inverses for these two maps are obtained as follows: regard the $n+1$-cube $I^{n+1}$ as $I^{n} \cup_{i}\left(I^{n} \times I\right)$ (the union being taken with respect to $i: I^{n} \times\{0\} \rightarrow I^{n}$ ), and suppose being a map $\theta$ : $K / K^{\prime} \times I^{n} \rightarrow p^{-1}(A)$ which maps $K / K^{\prime} \times I^{n}$ into $F_{h_{1}(b)}$. Hypothesis (c) permits an extension of $\theta$ to $K / K^{\prime} \times\left(I^{n} \cup{ }_{i}\left(I^{n} \times\left[0, \frac{1}{2}\right]\right)\right)$ such that $\theta \mid K / K^{\prime} \times\left(I^{n} \times\right.$ $\left.\left\{\frac{1}{2}\right\}\right)$ factors through $H_{1}$. Further extend $\theta$ to $K / K^{\prime} \times\left(I^{n} \cup_{i}\left(I^{n} \times I\right)\right) \rightarrow E$ using homotopy $H$. This, then, is the required inverse for $H_{1_{*}} \cdot h_{1_{*}}^{-1}$ is constructed by the same argument.

Definition 2.4. A $G$-triad $(X ; A, B)$ is said to be excisive if $X$ is the union of the interiors of $A$ and $B$. (All spaces here are assumed $G$-invariant.)

We have the following analogue of [Ma1, III.5.4].

THEOREM 2.5. Let $e:\left(X ; X_{1}, X_{2}\right) \rightarrow\left(Y ; Y_{1}, Y_{2}\right)$ be a map of excisive $G$-triads such that

$$
e:\left(X_{i} ; X_{1} \cap X_{2}\right) \rightarrow\left(Y_{i} ; Y_{1} \cap Y_{2}\right)
$$

is $a G-n$ equivalence for $i=1,2$.

Then $e:\left(X, X_{i}\right) \rightarrow\left(Y, Y_{i}\right)$ is also a $G-n$ equivalence.

Proof. One applies the adjunction $\mathcal{Q}\left(X, Y^{H}\right) \cong G \mathscr{U}(X \times G / H, Y)$ described in [Wa1] and observes that these functors preserve weak equivalence and excisiveness of triads, thus reducing the problem to the nonequivariant case, proved in [Ma1].

As a consequence, one obtains:

Lemma 2.6. Suppose that $\left(B ; B_{1}, B_{2}\right)$ is an excisive $G$-triad such that $p: E \rightarrow B$ is distinguished over $B_{1}, B_{2}$ and $B_{1} \cap B_{2}$. Then $p$ is a $G$-quasifibration.

Proof. This follows immediately from the long exact sequences of the $G$-triples $\left(B_{i}, B_{1} \cap B_{2}, b G\right)$ (for $i=1,2$ and $b \in B_{1} \cap B_{2}$ ) and their preimage triples, as well as those of $\left(B, B_{i}, b G\right)$ once 2.5 has been applied to the first set of $G$-triples. Compare [Ma1, III, §5].

Proposition 2.7. Let $p: E \rightarrow B$ be a $G$-map, and assume that $B$ has a $G$-filtration $\left\{F_{j} B\right\}$ such that

(a) $F_{0} B$ and each $G$-open subspace of $F_{j} B-F_{j-1} B$ are distinguished;

(b) For each $j>0$ there is an invariant open subset $U$ of $F_{j}(B)$, containing $F_{j-1}(B)$, together with $G$-homotopies $h_{t}: U \rightarrow U$ and $H_{t}: p^{-1}(U) \rightarrow p^{-1}(U)$, satisfying

(i) $h_{0}=1, h_{t} F_{j-1}(B) \subset F_{j-1}(B)$ and $h_{1}(U) \subset F_{j-1}(B)$.

(ii) $H_{0}=1$, and $H$ covers $h$.

(iii) $H_{1}$ restricts to a weak $K$-equivalence on fibers, where $K$ is the appropriate isotropy subgroup (as in Lemma 2.3).

Then $p$ is a $G$-quasifibration.

Proposition 2.7 follows by an easy induction argument using Lemmas 2.6 and 2.3. 
Finally, we describe the theory of gammaficaton in the equivariant context.

Notations 2.8. If $p: E \rightarrow B$ is a $G$-space over $B$, we may form a new $G$-space, $\Gamma_{p}: \Gamma E \rightarrow B$ by "gammafying" as in [Ma2, \$3.2]. (Briefly, we let $\Gamma E \subset \pi B \times E$ be the set of pairs $(\lambda, e)$, where $\lambda$ is a Moore path in $B$ starting at $p(e) . \Gamma_{p}$ is the end-point projection $\Gamma_{p}(\lambda, e)=\lambda(l(\lambda))$, where $\lambda(l)$ is the length of $\lambda$, so that $\lambda$ : $\overline{\mathbf{R}}^{+} \rightarrow B$ is constant for parameters $>l(\lambda)$.) There is also a based analogue $\Gamma^{\prime}$ of $\Gamma$ discussed there, and all of the following applies formally to based fibrations if we replace $\Gamma$ by $\Gamma^{\prime}$. Observe that $\Gamma E$ inherits a natural $G$-action such that $\Gamma_{p}$ is a $G$-map; in fact, a $G$-fibration with $\Gamma E G$-homotopy equivalent to $E$. (Similarly for $\Gamma^{\prime}$.)

We shall be needing the following lemma when constructing the transfer. It also serves as a useful illustration of the use of gammafication.

LemMa 2.9. Suppose that $p: E \rightarrow B$ is an equivariant fibration with fiber $F$ such that, if $\Lambda$ is the set of actions of closed subgroups of $G$ on $F$ relevant to $p$, then $F$ has the $H_{\alpha}$ homotopy type of a finite $H_{\alpha}-C W$ complex.

Suppose that we form a new space

$$
p^{\prime}: E^{\prime}=E \cup_{\phi^{\prime}}\left(\left(F \times_{H_{\alpha}} G\right) \times D^{n}\right) \rightarrow B \cup_{\phi^{\prime}}\left(G / H_{\alpha} \times D^{n}\right)=B^{\prime}
$$

by attaching a G-cell to $B$, covering the attachment by a G-map $\phi^{\prime}$ which is $a$ fiberwise equivariant homotopy equivalence (equivariant with respect to the relevant isotropy subgroups). Then $\Gamma_{p}^{\prime}: \Gamma E^{\prime} \rightarrow B^{\prime}$ is also a fibration whose fibers are equivariantly equivalent to $F$.

Proof. One observes that $p^{\prime}: E^{\prime} \rightarrow B^{\prime}$ is a $G$-quasifibration, and concludes that the fibers of $\Gamma E^{\prime}$ have the specified weak (equivariant) homotopy type, the passage from weak (equivariant) homotopy to strong equivariant homotopy being facilitated by Milnor's Theorem and its consequences (see [Wa1], as well as $\$ 5$ of this paper). A more detailed proof of a more general result appears in [Wa2].

Remark 2.10. One may replace $F \times{ }_{H} G$ by $F^{\prime} \times{ }_{H} G$ for some other compact space $F^{\prime}$ provided one is content to do without the comparison with a fixed fiber $F$. This point is discussed in relation to the classification theorem in [Wa2].

3. Equivariant duality and stable homotopy theory. The construction of equivariant Spanier-Whitehead duals for finite $G-C W$ complexes is due to Wirthmüller [Wi1] and is reintroduced here in the setting of an ambient $G$-space $G \mathbf{R}^{\infty}$. Let $G \mathcal{T}$ denote the category of based $G$-spaces and based maps.

Definition 3.1. A $G$-spectrum is a set of based $G$-spaces $\{E(V) \in G \mathcal{T}\}$ indexed by the $G$-subspaces of $G R^{\infty}$ together with structure maps

$$
\text { o: } \Sigma^{V} E(W) \rightarrow E(V+W) \text { for } V \perp W
$$

such that: 
(i) $\sigma=1$ when $V=\{0\}$;

(ii)

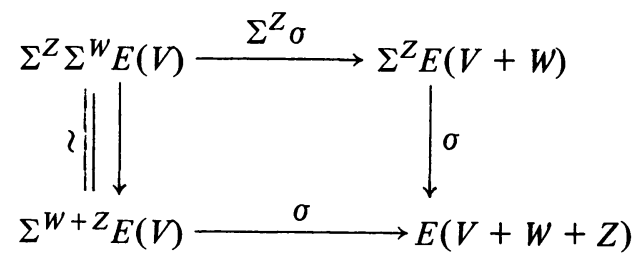

commutes when $V, W$, and $Z$ are pairwise orthogonal;

(iii) $\tilde{\sigma}: E(W) \rightarrow \Omega^{V} E(V+W)$ is a $G$-homeomorphism, where $\tilde{\sigma}$ is the adjoint of $\sigma$.

Maps of spectra are required to commute with the structure maps $\sigma$. Let $G \delta$ denote the category of $G$-spectra and maps of $G$-spectra.

Basis-free spectra were originally constructed by May (see, for example, [Ma1, §8]), and much of the theory easily goes over to the equivariant case by using $G \mathbf{R}^{\infty}$ as the ambient space.

Definition 3.2. Define functors $Q_{\infty}: G \mathcal{T} \rightarrow G \mathcal{S}$ and ( $)_{0}: G \mathcal{S} \rightarrow G \mathcal{T}$ by

$$
Q_{\infty}(X)(V)=\underset{W \perp V}{\operatorname{colim}} \Omega^{W} \Sigma^{V+W_{X}}
$$

and

$$
(E)_{0}=E(0)
$$

It is then formal [Ma1, §9] that the pair $\left(Q_{\infty},()_{0}\right)$ is an adjoint pair, and we have an adjunction

$$
G S\left(Q_{\infty} X, E\right) \cong G \mathcal{T}\left(X,(E)_{0}\right)
$$

Hence, by compactness, we have

$$
G \delta\left(Q_{\infty} X, Q_{\infty} Y\right) \cong \operatorname{colim} G \mathcal{T}\left(\Sigma^{V} X, \Sigma^{V} Y\right)
$$

whenever $X$ is compact, and it is easily verified that the colimit on the right-hand side is equivalent to the colimit colim $G \mathcal{T}\left(\Sigma^{\tilde{V}} X, \Sigma^{\tilde{V}} Y\right)$, where $\tilde{V}$ ranges through the category (made small) of all orthogonal $G$-modules, and direction is obtained from linear $G$-embeddings $\iota: \tilde{V} \rightarrow \tilde{W}$. (Note that (1) would not be possible if we were working in this cumbersome category instead of $G \mathbf{R}^{\infty}$.)

With the appropriate notion of homotopy, we obtain

$$
h G \delta\left(Q_{\infty} X, E\right) \simeq h G \mathcal{T}\left(X,(E)_{0}\right)
$$

and, for compact $X$,

$$
h G \delta\left(Q_{\infty} X, Q_{\infty} Y\right) \cong \operatorname{colim} h G \mathcal{T}\left(\Sigma^{V} X, \Sigma^{V} Y\right)
$$

by passage to homotopy categories, where the second equivalence displays the equivariant Spanier-Whitehead suspension category as the image subcategory under $Q_{\infty}$ of the category of compact spaces. A genuine stable $G$-homotopy category would be obtained from $h G \delta$ by formally inverting its weak equivalences, but we shall not enter into the requisite technicalities. Instead, we restrict attention to the suspension category of compact $G-C W$ complexes but retain the formal language of spectra where convenient. 
DefinItion 3.3. As in [Wi1, §1], we define a $G$-duality to be a $G$-map $u$ : $Q_{\infty} S^{V} \rightarrow Q_{\infty}\left(\Sigma^{W}(X \wedge \hat{X})\right)$ such that $u$ induces isomorphisms

$$
u /: h K \delta\left(Q_{\infty} S^{*}, X\right) \rightarrow h K \delta\left(\hat{X}, Q_{\infty} S^{*}\right)
$$

and

$$
u^{*} /: h K S\left(Q_{\infty} S^{*}, \hat{X}\right) \rightarrow h K \delta\left(X, Q_{\infty} S^{*}\right)
$$

$u$ is then called an $\alpha-G$ duality, where $\alpha=V-W \in R O(G)$. Note that this defines $\hat{X}$ uniquely up to $G$-homotopy [Wi1].

Observe that by 3.2 , we may represent such duality maps by elements of colim $h G \tau\left(S^{V+L}, \Sigma^{W+L}(X \wedge \hat{X})\right)=\pi_{\alpha}^{S}(X \wedge \hat{X})$ where $\pi_{\alpha}^{S}$ denotes the $\alpha$ th equivariant stable homotopy group. The results in [Wi1] easily imply that:

Proposition 3.4. Let $u: S^{\alpha *} \rightarrow X \wedge \hat{X}^{*}$ represent an $\alpha-G$ duality. The asterisks indicate that the map is in some suspension $\Sigma^{V}$. Then $X \wedge \hat{X}$ is self-dual via

$$
\mu: S^{2 \alpha *} \stackrel{u \wedge u}{\rightarrow} X \wedge \hat{X} \wedge X \wedge \hat{X}^{*} \stackrel{(1,3)}{\rightarrow} X \wedge \hat{X} \wedge X \wedge \hat{X}^{*}
$$

where $(1,3)$ permutes the first and third coordinates.

Definition 3.5. Let $f: X \rightarrow X$ be a $G$-map and let $X$ have an $\alpha-G$ dual $\hat{X}$ for some $\alpha \in R O(G)$. Then define the equivariant reduced Lefshetz number by $\tilde{\Lambda}(f)=$ $\theta\left(f_{S}\right) \in A(G)$, the Burnside ring of $G$, where $f_{S}$ is the composite:

$$
S^{\alpha *} \underset{u}{\rightarrow} X \wedge \hat{X}^{*} \underset{f \wedge 1}{\rightarrow} X \wedge \hat{X}^{*} \underset{\hat{u}}{\rightarrow} S^{\alpha *}
$$

in $\pi_{0}^{S}\left(S^{9}\right), \hat{u}$ being dual to $u$ via a duality $\mu$, and where $\theta$ is the isomorphism constricted by tom Dieck [Di1], from $\pi_{0}^{S}\left(S^{0}\right)$ to $A(G)$.

Observe that, if $H \subset G$ is a closed subgroup, then $\tilde{\Lambda}\left(f^{H}\right)$ is the usual reduced Lefshetz number (see [Di1]). (The fact that $\mu^{H}$ is a duality for any $H<G$ and $G$-duality $\mu$ stems from the fact that $G$-duals of orbits are their Thom spaces in orthogonal representations.)

4. Equivariant fiberwise duality. The construction of fiberwise duality is due to Becker and Gottlieb [Be1], and is generalized here to $(G \mathscr{F}, F)$ fibrations for suitable categories $(G \mathscr{F}, F)$. Recall the notion of a based $G$-space $E$ over $B$ (see \$4.1).

Lemma 4.1. Let $E$ and $E^{\prime}$ be based $G$-spaces over $B$, and let $f: E \rightarrow E^{\prime}$ be $a$ section-preserving $G$-map over $B$. Let $C_{\mathscr{G}} E, C_{\mathscr{G}} f$ and $\Sigma_{\mathscr{F}} E$ denote, respectively, the fiberwise reduced cone of $E$, the fiberwise cofiber, $C_{\Phi} E \cup_{f} E^{\prime}$, of $f$, and the fiberwise suspension of $E$. Then the natural maps induce for any $E^{\prime \prime}$, an exact sequence

$$
\cdots\left\{\Sigma_{\mathscr{F}} E, E^{\prime \prime}\right\}_{\mathscr{F}} \rightarrow\left\{C_{\mathscr{F}} f, E^{\prime \prime}\right\}_{\mathscr{F}} \rightarrow\left\{E^{\prime}, E^{\prime \prime}\right\}_{\mathscr{F}} \rightarrow\left\{E, E^{\prime \prime}\right\}_{\mathscr{F}} \rightarrow \cdots
$$

of abelian groups, where $\{X, Y\}_{\mathscr{F}}$ denotes the set colim $\left[\Sigma_{\mathscr{F}}^{V} X, \Sigma_{\mathscr{F}}^{V} Y\right]_{\mathscr{F}}$ of fiberwise homotopy classes of maps over $B$ (taken over $V \subset G \mathbf{R}^{\infty}$ ). Here, $\Sigma_{\mathscr{G}}^{V} X=S^{V} \wedge_{\mathscr{F}} X$, where $\wedge_{\mathcal{F}}$ is fiberwise smash product.

Proof. For each fixed $V$, exactness is a formal consequence of the definitions, and the passage to colimits is automatic since $\Sigma_{\mathscr{F}}^{U}$ commutes with $C_{F}$ and $\Sigma_{\mathscr{F}}$ 
LemmA 4.2. Let $p: E \rightarrow B$ be a based $G$-space, and suppose that $(B, A)$ is $a$ $G-N . D . R$. pair in $G \mathscr{Q}$ such that there is a G-retraction $r: B \times I \rightarrow B \times\{0\} \cup A \times$ $I$ which is covered by a G-retraction

$$
\tilde{r}: E \times I \rightarrow E \times\{0\} \cup p^{-1}(A) \times I,
$$

the target being the fiberwise reduced mapping cylinder. Let $p^{\prime}: E^{\prime} \rightarrow B$ be a based $G$-fibration. Then the natural map $C_{\oiint} \tilde{i} \rightarrow E / p^{-1}(A)$ induces an isomorphism

$$
\left[C_{\mathscr{F}} \tilde{i}, E^{\prime}\right]_{\mathscr{F}} \cong\left[E / p^{-1}(A), E^{\prime}\right]_{\mathscr{F}}
$$

where $E / p^{-1}(A)$ is the $G$-space over $B$ obtained by collapsing each fiber over $A$ to its basepoint, and $\tilde{i}: p^{-1}(A) \cup{ }_{\sigma} B \rightarrow E$ is the inclusion, $\sigma: A \rightarrow p^{-1}(A)$ being the restriction of the section of $p$.

Proof. Suppose given $f: C_{\mathcal{G}} \tilde{i} \rightarrow E^{\prime}$ over $B$. We have the following diagram of G-maps:

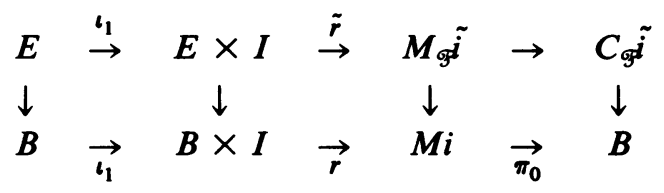

in which $\iota_{1}(x)=(x, 1), \tilde{r}$ and $r$ are as in the hypothesis, and $M_{\mathscr{F}}$ and $M$ are, respectively, the fiberwise and ordinary reduced mapping cylinder functors. ( $i$ : $A \rightarrow B$ is the inclusion.)

This diagram factors to give:

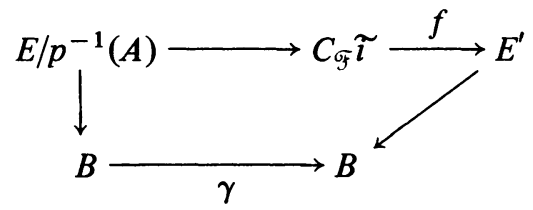

where $\gamma=\pi_{0} r \iota_{1}$. But $\gamma$ is $G$-homotopic to the identity, and the $G$-CHP gives a map $E / p^{-1}(A) \rightarrow E^{\prime}$ as required. The verification that this defines the required inverse is straightforward.

Definition 4.3. Let $p: E \rightarrow B$ be a based $G$-fibration. A fiberwise $G$-duality is a map

$$
u: S^{V} \times B \rightarrow E \wedge \wedge_{\mathscr{F}} \hat{E}
$$

of based $G$-fibrations over $B$ such that for each $x \in B, u$ restricts to a $G_{x}$ duality $S^{V} \rightarrow F_{x} \wedge \hat{F}_{x}$ over $x$, where $F_{x}=p^{-1}(x)$.

LEMma 4.4. Let $u: S^{V} \times B \rightarrow E \wedge_{\mathscr{F}} \hat{E}$ be a fiberwise G-dualtiy. Then for all based $G$-fibrations $X$ and $Y$ over $B$, we have

$$
D u:\left\{X, Y \wedge_{\mathscr{F}} E\right\}_{\mathscr{F}} \simeq\left\{X \wedge_{\mathscr{F}} \hat{E}, Y\right\}_{\mathscr{F}}
$$

whenever $B$ is a finite dimensional $G-\mathrm{CW}$ complex.

Proof. This is proved by induction over the skeleta of $B$.

If $B$ is an orbit, $B=G / H$, the result is an immediate consequence of the theory 
of equivariant duality [Wi1] since any map over $G / H$ is entirely specified by its restriction to the fiber over the identity coset. Similarly for disjoint unions of $G$-orbits.

Suppose then that the lemma is valid for all $n-1$ dimensional $G-C W$ complexes, and let $B$ be an $n$-dimensional $G-C W$ complex. Then, considering parts over $B^{n-1}, u \mid S^{V} \times B^{n-1}$ sets up an isomorphism

$$
D u \mid:\left\{X\left|B^{n-1}, Y\right| B^{n-1} \wedge_{\mathscr{F}} E \mid B^{n-1}\right\}_{\mathscr{F}} \cong\left\{X\left|B^{n-1} \wedge_{\mathscr{F}} \hat{E}\right| B^{n-1}, Y \mid B^{n-1}\right\}_{\mathscr{F}}
$$

over $B^{n-1}$.

Now apply the 5-lemma to the sequence:

$$
\begin{gathered}
\left\{\frac{X \mid B^{n}}{X \mid B^{n-1}}, \frac{Y\left|B^{n} \wedge_{\mathscr{F}} E\right| B^{n}}{Y\left|B^{n-1} \wedge_{\mathscr{F}} E\right| B^{n-1}}\right\}_{\mathscr{F}} \\
\| \leftarrow(\text { since maps are fiberwise }) \\
\left\{\frac{X \mid B^{n}}{X \mid B^{n-1}}, Y\left|B^{n} \wedge_{\mathscr{F}} E\right| B^{n}\right\}_{\mathscr{F}} \\
\downarrow \\
\left\{X\left|B^{n-1}, Y\right| B^{n} \wedge_{\mathscr{F}} E \mid B^{n}\right\}_{\mathscr{F}} \\
\downarrow \\
\left\{X\left|B^{n-1}, Y\right| B^{n-1} \wedge_{\mathscr{F}} E \mid B^{n-1}\right\}_{\mathscr{F}}
\end{gathered}
$$

of spaces over $B^{n}$, where spaces over $B^{n-1}$ are regarded as being over $B^{n}$ by using the section to attach a copy of $B^{n}$. Here,

$$
\frac{X \mid B^{n}}{X \mid B^{n-1}} \text { and } \frac{Y\left|B^{n} \wedge_{\mathscr{G}} E\right| B^{n}}{Y\left|B^{n-1} \wedge_{\mathscr{F}} E\right| B^{n-1}}
$$

are $G$-fiberwise homotopy equivalent to trivial $G$-fibrations of the form

$$
\amalg_{H_{\alpha}} F \times H_{H_{\alpha}} G \times \dot{D}^{n} \rightarrow{\underset{H}{\alpha}}_{H_{\alpha}} G / H_{\alpha} \times \dot{D}^{n} .
$$

This proves the inductive step.

COROllary 4.5. $E \wedge_{\mathscr{g}} \hat{E}$ is self-dual via the map

$$
\mu: B \times S^{2 V} \stackrel{u \wedge u}{\rightarrow} E \wedge_{\mathscr{F}} \hat{E} \wedge_{\mathscr{F}} E \wedge_{\mathscr{F}} \hat{E} \stackrel{(1,3)}{\rightarrow} E \wedge_{\mathscr{F}} \hat{E} \wedge_{\mathscr{F}} E \wedge_{\mathscr{F}} \hat{E}
$$

whenever $B$ is finite dimensional.

THeORem 4.6. Let $p: E \rightarrow B$ be a based $G$-fibration with fiber $F$ such that $B$ is a finite dimensional G-CW complex, and such that $F$ has the $H_{\alpha}$ homotopy type of $a$ finite $H_{\alpha}-\mathrm{CW}$ complex for each action of each subgroup $H_{\alpha}$ on $F$ appearing (up to $H_{\alpha}$-homotopy) in $E$. Then $p$ has a fiberwise $G$-dual $\hat{p}: \hat{E} \rightarrow B$.

Proof. We proceed by induction on the skeleta of $B$, and we let $E_{i}=p^{-1}\left(B^{i}\right)$ and $p_{i}=p \mid E_{i}$. By consideration of disjoint unions, the result will hold for $B^{0}$ if it does so when $B^{0}=G / H$ for some closed subgroup $H$ of $G$. Then $p_{0}: E_{0} \rightarrow B^{0}$ is of the form $p: F \times{ }_{H} G \rightarrow G / H$. Choose a duality $S^{V} \rightarrow F \wedge \hat{F}$ of $H$-spaces for $V \subset H \mathbf{R}^{\infty}$. By the Frobenius Property, there exists an $H$-isometry $W+V \rightarrow G \mathbf{R}^{\infty}$ with $W \perp V$ and $H$-invariant. 
Now $S^{V+W} \rightarrow F \wedge \Sigma^{W} \hat{F}$ is also a duality of $H$-spaces, where $S^{V+W}$ may be regarded as the compactification of a subspace of $G \mathbf{R}^{\infty}$.

Twisting both sides with $G$, we obtain a map

$$
\begin{aligned}
& S^{V+W} \times G / H \cong S^{V+W} \times{ }_{H} G \rightarrow\left(F \wedge \Sigma^{W} F\right) \times_{H} G \\
&\|) \\
&\left(F \times_{H} G\right) \wedge_{\overline{\mathrm{y}}}\left(\Sigma^{W} \hat{F} \times_{H} G\right)
\end{aligned}
$$

where the first isomorphism is the map $(s,[g]) \mapsto\left[s g^{-1}, g\right]$, and all the components are fiber-preserving maps. Clearly, this map is a fiberwise $G$-duality for $G / H=$ $B^{0}$.

Suppose then that we have constructed a fiberwise $G$-duality for $E_{n}$, and that $B$ is $n+1$-dimensional. We may as well assume that $B=B^{n} \cup{ }_{\phi} G / H \times D^{n-1}$, as the argument will show.

We have the following pullback diagrams in some fiberwise suspension by a $G$-module.

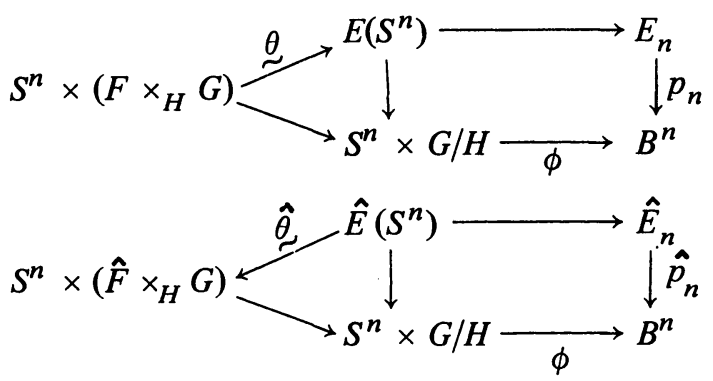

Here, $\hat{E}\left(S^{n}\right)=E\left(S^{n}\right)$, where $E\left(S^{n}\right)=\phi^{*}\left(p_{n}\right), \theta$ is a fiberwise $G$-equivalence, and $\hat{\theta}$ is dual to $\theta$ relative to a fixed duality $\mu^{\prime}$ for $F$ and the pullback $\tilde{\mu}$ of a duality $\mu$ for $E$. The situation is now displayed as follows (in some fiberwise suspension):

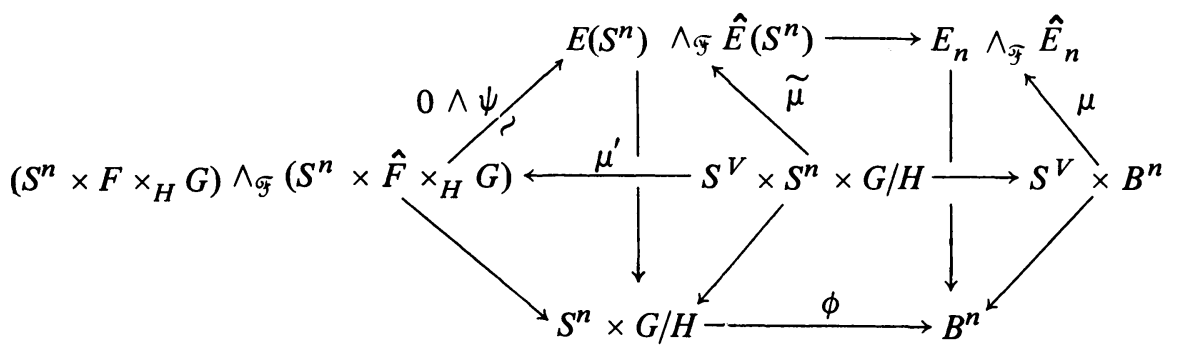

Here, the top face of one tetrahedron on the left $G$-homotopy commutes over $S^{n} \times G / H$, and the rest of the diagram strictly commutes. $\psi$ is a fiberwise $G$-homotopy inverse to $\hat{\theta}$ ( $\theta$ being a fiberwise equivalence).

The construction of the required duality is then explained by the following diagram, again in some suspension by a $G$-module. 


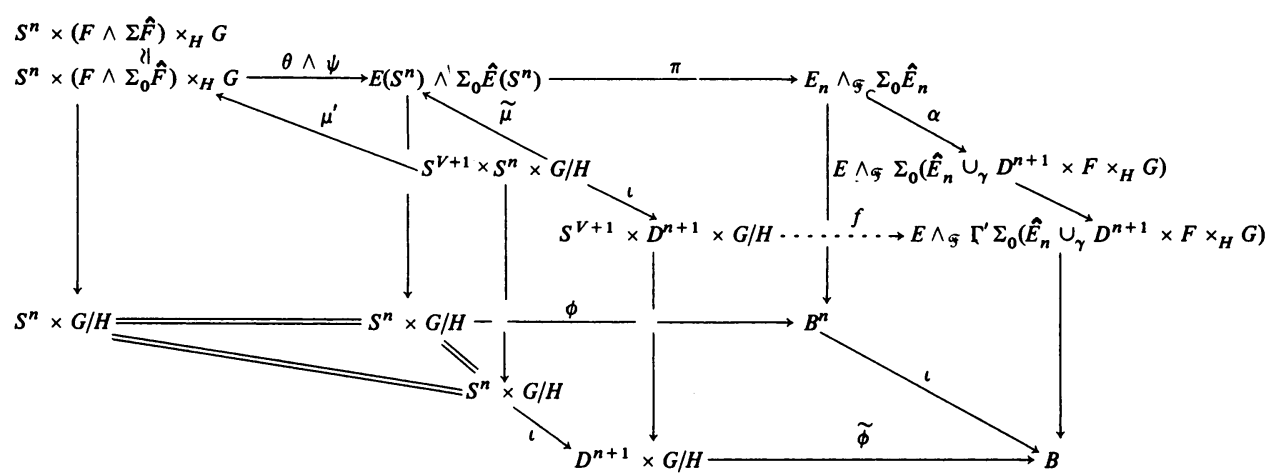

Here, $\Sigma_{0}$ denotes unreduced (based) suspension,

$$
\gamma=\pi \circ \phi: S^{n} \times\left(\Sigma_{0} \hat{F} \times_{H} G\right) \rightarrow \Sigma_{0} E\left(S^{n}\right) \rightarrow \Sigma_{0} \hat{E}_{n},
$$

$\tilde{\phi}$ retracts one outer rim of $D^{n+1}$ to $S^{n}$, and the diagram commutes, except possibly for the upper face of the tetrahedron on the left, which homotopy commutes over $S^{n} \times G / H . \Gamma^{\prime}$ is the functor discussed in [Ma2, 3.2]. Existence of the map $f$ follows from the fact that $\alpha \circ \pi \circ(\theta \wedge \psi)$ is null-homotopic, and from the homotopy $\theta \wedge \psi \circ \mu^{\prime} \sim \tilde{\mu}$. Let $E=\Gamma^{\prime} \Sigma_{0}\left(\hat{E}_{n} \cup{ }_{\gamma} D^{n} \times F \times{ }_{H} G\right)$. Then $f$ and the duality $\mu$ fit together to give a map

$$
\rho: S^{V+1} \times B \rightarrow E \wedge{ }_{\mathscr{F}} \hat{E}
$$

such that the diagram

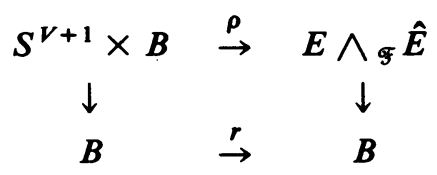

commutes, where $r$ deforms the outer rim of the $n+1$ cell into the boundary. Also, $\rho$ restricts to a duality over each point $b \in B$.

Since $r \sim 1$, we may find a map $\mu: S^{V+1} \times B \rightarrow E \wedge_{\mathscr{F}} \hat{E}$ over $B$ by the $G-C H P$. Since the property of being a fiberwise duality is preserved by such homotopies, it follows that $\mu$ is a fiberwise duality, as required.

5. Equivariant transfer. Once equivariant fiberwise duality is known to exist, the definition and properties of equivariant transfer generalize fairly easily from the nonequivariant case.

DEFINITION 5.1. Let $p: E \rightarrow B$ be an unbased fibration with fiber compact $F$ over a finite dimensional $G-C W$ complex.

By the equivariant transfer associated with a $G$-map $f: E \rightarrow E$ over $B$, we mean the map $t_{f}: Q_{\infty} B^{+} \rightarrow Q_{\infty} E^{+}$defined by the following commutative diagram:

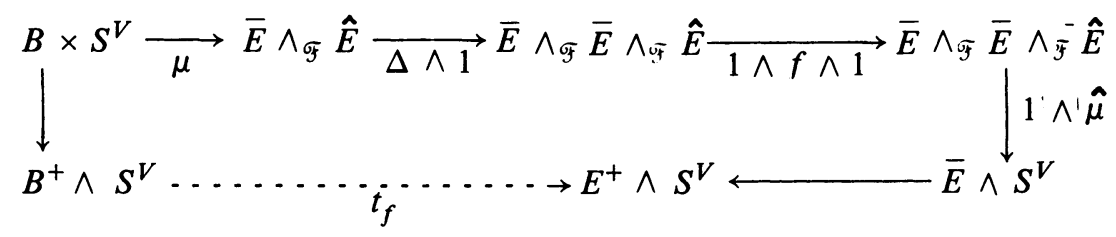


where $\mu$ and $\hat{\mu}$ are a $G$-dual pair of $G$-duality maps, $\bar{E}=E \amalg B, \hat{E}$ is fiberwise $G$-dual to $\bar{E}$, and $\Delta$ is the diagonal.

Observe that this composite defines a unique class in $h G S\left(Q_{\infty} B^{+}, Q_{\infty} E^{+}\right)$ (independently of choice of dualities), and that the formal arguments in [Be1] go over to the equivariant case to give us the following naturality properties:

Proposition 5.2. (i) Let $f_{i}: E_{i} \rightarrow E_{i}(i=1,2)$ be $G$-maps over $B$ and let $h$ : $E_{1} \rightarrow E_{2}$ be a fiberwise G-homotopy equivalence such that $h \circ f_{1}=f_{2} \circ h$. Then $t_{f_{2}} \sim h t_{f_{1}}$.

(ii) (Naturally over pullbacks). If $g: B^{\prime} \rightarrow B$ is a $G$-map, then the following diagram G-homotopy commutes:

$$
\begin{array}{cccc}
Q_{\infty} E^{\prime+} & \rightarrow & Q_{\infty} E^{+} \\
t_{f} \uparrow & & t_{f} \\
Q_{\infty} B^{\prime+} & \vec{Q}_{\infty} g^{+} & Q_{\infty} B^{+}
\end{array}
$$

where $p^{\prime}: E^{\prime} \rightarrow B^{\prime}$ is the induced fibration $g^{*}(p)$ and $f^{\prime}=g^{*}(f)$.

(iii) If $p: E \rightarrow B$ and $p^{\prime}: E^{\prime} \rightarrow B^{\prime}$ are suitable $(G \mathscr{F}, F)$ fibrations, then the composite

$$
\begin{array}{ccc}
\Sigma^{2 V}\left(B \times B^{\prime}\right)^{+} \cong & \Sigma^{V} B^{+} \wedge \Sigma^{V} B^{+} \\
& & \downarrow t_{f} \wedge t_{f^{\prime}}^{\prime} \\
\Sigma^{2 V}\left(E \times E^{\prime}\right)^{+} \cong & \Sigma^{V} E^{+} \wedge \Sigma^{V} E^{\prime+}
\end{array}
$$

represents $t_{f \wedge f^{\prime}}$

(iv) Passing to generalized G-cohomology theories, transfer defines a morphism of graded modules over the cohomology of $B$ (itself an algebra over $A(G)$ ).

Part (iv) requires a little explanation: Recall that an additive $G$-cohomology theory defined on based $G-\mathrm{CW}$ complexes is an $R O(G)$-graded contravariant group-valued functor $K^{*}$ which converts $G$-cofibration sequences into long exact sequences and which are equipped with natural suspension isomorphisms of the form

$$
\sigma^{V}: K^{\alpha+V}\left(\Sigma^{V} X\right) \simeq K^{\alpha}(X)
$$

for $\alpha \in R O(G)$. It is a formal consequence of Brown's Theorem (see, for example, [Ma1, §2]) that such theories may be represented by " $\Omega G$-prespectra" indexed on $R O(G)$ for which the structural maps $\sigma$ exist (in the category $G \mathcal{T}$ ) but for which the $\tilde{\sigma}$ need only be weak $G$-equivalences (and hence $G$-equivalences by $G$-Milnor's Theorem in the [Wa1]). The algebra structure over $A(G)$ is then given by

$$
\Sigma^{U} \Sigma^{V} B^{+} \stackrel{g \wedge 1}{\rightarrow} \Sigma^{U} \Sigma^{V} B^{+} \stackrel{\Sigma^{U_{f}}}{\rightarrow} \Sigma^{U} T(W) \rightarrow T(U+W)
$$

where $g: S^{U} \rightarrow S^{U}$ represents a typical element of $A(G)$ and $f: \Sigma^{V} B^{+} \rightarrow T(W)$ represents a typical element of the cohomology of $B$. Associativity of this structure follows from associativity of the suspension functors, and all the usual coherence is formal.

The basic result about equivariant transfer is given by the following theorem, and, in a more accessible form, in its successor. 
Theorem 5.3. Let $p: E \rightarrow B$ and $f: E \rightarrow E$ be as above, with $B$ G-connected, and suppose that $H^{*}$ is an equivariant cohomology theory with a weak dimension axiom (i.e., such that $H^{0}(i): H^{0}(X) \rightarrow H^{0}(*) \cong A(G)$ is an isomorphism for G-connected $X)$. Then $H^{*}\left(p \circ t_{f}\right)(x)=\Lambda(f)_{x}$, where $\Lambda(f)$ is defined to be $\tilde{\Lambda}\left(f^{+}\right)$.

Proof. The definition of $\tilde{\Lambda}\left(f^{+}\right)$and of $t_{f}$ shows this to be true when $X=*$ (whether or not we insist that $H^{0}(i)$ be an isomorphism in the hypothesis), and the result follows by Proposition 5.2 and the remarks following it.

Clearly, the $G$-connectivity requirement on $B$ is unreasonably strong in general, as we are asking that all the fixed point sets of $B$ be connected. However, we may specialize to fixed point sets quite easily as follows:

THEOREM 5.4. Let $p: E \rightarrow B$ and $f: E \rightarrow E$ be as above, with $B^{H}$ connected for some closed subgroup $H$ of $G$. Then, if $H^{*}$ denotes any cohomology theory with dimension axiom (as in 5.3, but with $Z$ replacing $A(G)$ and equivariance dropped), then $H^{*}\left(p^{H} \circ t_{f}^{H}\right)(x)=\Lambda\left(f^{H}\right) x$.

Proof. This is obvious from the definitions and 5.3, or by direct verification that $t_{f}^{H}$ is the nonequivariant transfer for $p^{H}$ and $f^{H}$.

6. Equivariant cellular theory and fibrations. Here, we obtain the equivariant version of a theorem due to Stasheff [St1].

TheOREM 6.1. Let $p: E \rightarrow B$ be a $G$-fibration with fiber $F$, and assume that $B$ has the G-homotopy type of a countable G-CW complex. (That is, $B \in G \mho_{0}$ in the notation of [Wa1].) Then $E \in G W_{0}$ if and only if $F \in H_{\alpha} W_{0}$ for each action $\alpha$ on $F$ appearing (up to $H_{\alpha}$ homotopy) in $E$.

Proof. Half of this theorem (the "only if" part) is proved in [Wa1] by replacing $p$ by $\Gamma p$. The converse is a generalization of a result by Stasheff [St1] as proved by Schön [Sc1] (his proof being simpler than the original proof of Stasheff).

Recall from [Wa1] that we may replace $E$ by a $G-C W$ approximation $W E$.

Let $\gamma: W E \rightarrow E$ be a $G-C W$ approximation where $W E$ may be assumed countable by countability of the $G$-homotopy groups, the number of subgroup types, and the long exact fibration sequences. We may replace $\gamma$ by an equivariant fibration $\Gamma \gamma: \Gamma W E \rightarrow E$. Let $p^{\prime}: \Gamma W E \rightarrow B$ be the $G$-map $p \circ \Gamma \gamma$. Then the following diagram commutes:

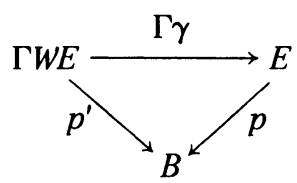

Further, $p^{\prime}$ and $p$ are both equivariant fibrations where the fibers of $p^{\prime}$ are in $H W_{0}$ for the appropriate subgroups $H$, by [Wa1].

Now $\Gamma \gamma$ is a weak-G-equivalence, and the result follows by the long exact fibration sequences for $p$ and $p^{\prime}$, the five lemma, the Equivariant Whitehead Theorem [Wa1], and $G$-Dold's Theorem. 


\section{BIBLIOGRAPHY}

[Be1] J. C. Becker and D. G. Gottlieb, Transfer and duality, Purdue Univ. (preprint).

[Di1] T. tom Dieck, The Burnside ring and equivariant stable homotopy, University of Chicago, Chicago, Ill., 1974 (mimeographed notes).

[Ma1] J. P. May, Homotopic foundations of algebraic topology, University of Chicago, Chicago, Ill., (mimeographed notes).

[Ma2] _, Classifying spaces and fibrations, Mem. Amer. Math. Soc. No. 155, 1975.

[MHW] J. P. May, H. Hauschild and S. Waner, Equivariant infinite loop spaces (in preparation).

[Mi1] J. Milnor, On spaces having the homotopy type of a CW complex, Trans. Amer. Math. Soc. 90 (1959), 272-280.

[Ni1] G. Nishida, On the equivariant J-groups and equivariant stable homotopy types of representations of finite groups, Kyoto (preprint).

[Sc1] R. Schön, Fibrations over a CWh-base, Proc. Amer. Math. Soc. 62 (1977), 165-166.

[St1] J. D. Stasheff, A classification theorem for fibre spaces, Topology 2 (1963), 239-246.

[Wa1] S. Waner, Equivariant homotopy theory and Milnor's Theorem, Trans. Amer. Math. Soc. 258 (1980), 351-368.

[Wa2] __ Classification of equivariant fibrations, Trans. Amer. Math. Soc. (to appear)

[W23] __ The equivariant approximation theorem, Princeton Univ. (preprint).

[Wa4] _ Cyclic group actions and the Adams conjecture, Princeton Univ. (preprint).

[Wi1] K. Wirthmüller, Equivariant S-duality, Arch. Math. 26 (1975), 427-431.

Department of Mathematics, Princeton University, Prnnceton, New Jersey 08540 